\title{
Subspace Distinguisher for 5/8 Rounds of the ECHO-256 Hash Function ${ }^{\star}$
}

\author{
Martin Schläffer \\ Institute for Applied Information Processing and Communications (IAIK) \\ Graz University of Technology, Inffeldgasse 16a, A-8010 Graz, Austria \\ martin.schlaeffer@iaik.tugraz.at
}

\begin{abstract}
In this work we present first results for the hash function of ECHO. We provide a subspace distinguisher for 5 rounds and collisions for 4 out of 8 rounds of the ECHO-256 hash function. The complexities are $2^{96}$ compression function calls for the distinguisher and $2^{64}$ for the collision attack. The memory requirements are $2^{64}$ for all attacks. To get these results, we consider new and sparse truncated differential paths through ECHO. We are able to construct these paths by analyzing the combined MixColumns and BigMixColumns transformation. Since in these sparse truncated differential paths at most one fourth of all bytes of each ECHO state are active, missing degrees of freedom are not a problem. Therefore, we are able to mount a rebound attack with multiple inbound phases to efficiently find according message pairs for ECHO.
\end{abstract}

Keywords: hash functions, SHA-3 competition, ECHO, cryptanalysis, truncated differential path, rebound attack, subspace distinguisher, near-collisions, collision attack.

\section{Introduction}

Many new and interesting hash function designs have been proposed in the NIST SHA-3 competition [17. In this paper, we analyze the hash function ECHO [1, which is one of 14 Round 2 candidates of the competition. ECHO is a wide-pipe, AES based design which transforms 128-bit words similar as AES transforms bytes. Inside these 128-bit words, two standard AES rounds are used. So far, most cryptanalytic results of ECHO were limited to the internal permutation [13, 7 . Recently, reduced round attacks on the wide-pipe compression function of ECHO have been published in [18, which cover up to 4/8 rounds for ECHO-256 and 6/10 rounds of ECHO-512. However, a drawback of attacks on building blocks (such as compression functions or permutations) is that they cannot be used to compare SHA-3 candidates due to their great design variety and different requirements for building blocks.

Therefore, in this work we analyze the hash function of ECHO and present results for up to $5 / 8$ rounds of ECHO-256. We use the subspace distinguisher [9, 10]

\footnotetext{
* Near-collisions for 4.5/8 rounds of the hash function and compression function results for $7 / 8$ rounds without chosen salt are given in an extended version of this paper 19 .
}

A. Biryukov, G. Gong, and D.R. Stinson (Eds.): SAC 2010, LNCS 6544, pp. 369-387, 2011.

(C) Springer-Verlag Berlin Heidelberg 2011 
to compare our distinguishing attacks with the generic complexity on ideal hash functions. Our results greatly improve upon previously results, which were attacks on a similar number of rounds of the compression function 18 . Furthermore, attacks on the compression function for up to $7 / 8$ rounds of ECHO-256 and 7/10 rounds of ECHO-512 are given in an extended version of this paper [19]. The main improvement is to consider a new type of sparse truncated differential paths by placing only a single active byte in the ECHO state with 16 active AES states. In all previous paths, the full active ECHO states also had full active AES states. The construction of such paths is possible by combining the last MixColumns transformation of the second AES round with the BigMixColumns transformation of an ECHO round to a SuperMixColumns transformation.

The attack itself is a rebound attack [14] with multiple inbound phases. Similar attacks have been applied to the SHA-3 candidate LANE 12 and the hash function Whirlpool 9]. Since the truncated differential paths are very sparse, we have plenty degrees of freedom to merge the solutions of these multiple inbound phases. Note that using multiple inbound phases, we can control more distant parts of much longer truncated differential paths than in a start-from-the-middle attack [13] or simple Super-Sbox analysis 9 15.7] where the controlled rounds are limited to only the middle rounds. To merge independent solutions of multiple inbound phases, we use a technique based on the generalized birthday attack [20].

\section{Description of ECHO}

In this section we briefly describe the AES based SHA-3 candidate ECHO. For a detailed description of ECHO we refer to the specification [1. Since ECHO heavily uses AES rounds, we describe the AES block cipher first.

\subsection{The AES Block Cipher}

The block cipher Rijndael was designed by Daemen and Rijmen and standardized by NIST in 2000 as the Advanced Encryption Standard (AES) [16. The AES follows the wide-trail design strategy [4,5] and consists of a key schedule and state update transformation. Since ECHO does not use the AES key schedule, we just describe the state update here.

In the AES, a $4 \times 4$ state of 16 bytes is updated using the following 4 round transformations, with 10 rounds for AES-128. The non-linear layer SubBytes (SB) applies the AES S-Box to each byte of the state independently. The cyclical permutation ShiftRows (SR) rotates the bytes of row $j$ to the left by $j$ positions. The linear diffusion layer MixColumns (MC) multiplies each column of the state by a constant MDS matrix $M_{\mathrm{MC}}$. AddRoundKey (AK) adds the 128-bit round key $K_{i}$ to the AES state. Note that a round key is added prior to the first round and the MixColumns transformation is omitted in the last round of AES. For a detailed description of the AES we refer to [16]. 


\subsection{The ECHO Hash Function}

The ECHO hash function is a SHA-3 candidate submitted by Benadjila et al. 1]. It is a double-pipe, iterated hash function and uses the HAIFA [2] domain extension algorithm. More precisely, a padded $t$-block message $M$ and a salt $s$ are hashed using the compression function $f\left(H_{i-1}, M_{i}, c_{i}, s\right)$, where $c_{i}$ is a bit counter, IV the initial value and $\operatorname{trunc}\left(H_{t}\right)$ a truncation to the final output hash size of $n$ bits:

$$
\begin{aligned}
H_{0} & =I V \\
H_{i} & =f\left(H_{i-1}, M_{i}, c_{i}, s\right) \quad \text { for } 1 \leq i \leq t \\
h & =\operatorname{trunc}_{n}\left(H_{t}\right) .
\end{aligned}
$$

The message block size is 1536 bits for ECHO-256 and 1024 bits for ECHO-512, and the message is padded by adding a single 1 followed by zeros to fill up the block size. Note that the last 18 bytes of the last message block always contain the 2-byte hash output size, followed by the 16-byte message length.

The compression function of ECHO uses one internal 2048-bit permutation $P$ which manipulates 128-bit words similar as AES manipulates bytes. The permutation consists of 8 rounds in the case of ECHO-256 and has 10 rounds for ECHO-512. The internal state of the permutation $P$ can be modeled as a $4 \times 4$ matrix of 128-bit words. We denote one ECHO state by $S_{i}$ and each 128-bit word or AES state is indexed by $[r, c]$, with rows $r \in\{0, \ldots, 3\}$ and columns $c \in\{0, \ldots, 3\}$ of the ECHO state.

The 2048-bit input of the permutation (which is also tweaked by the counter $c_{i}$ and salt $s$ ) are the previous chaining variable $H_{i-1}$ and the current message block $M_{i}$, concatenated to each other. After the last round of the permutation, a feed-forward (FF) is applied to get the preliminary output $V$ :

$$
V=P_{c_{i}, s}\left(H_{i-1} \| M_{i}\right) \oplus\left(H_{i-1} \| M_{i}\right) .
$$

To get the 512-bit chaining variable $H_{i}$ for ECHO-256, all columns of the ECHO output state $V$ are XORed. In the case of ECHO-512, the 1024-bit chaining variable $H_{i}$ is the XOR of the two left and the two right columns of $V$. The feed-forward together with the compression of columns is called the BigFinal (BF) operation. To get the final output of the hash function, the lower half is truncated in the case of ECHO-256 and the right half is truncated for ECHO-512.

The round transformations of the ECHO permutation are very similar to AES rounds, except that 128-bit words are used instead of bytes. One round is the composition of the following three transformations in the given order: The nonlinear layer BigSubWords (BSW) applies two AES rounds to each of the 16 128bit words of the internal state. The first round key consists of a counter value initialized by $c_{i}$ and increased for every AES state and round of ECHO. The second round key consists of the 128-bit salt $s$. The cyclical permutation BigShiftRows (BSR) rotates the 128-bit words of row $j$ to the left by $j$ words. The linear diffusion layer BigMixColumns (BMC) mixes the AES states of each ECHO column by the same MDS matrix $M_{\mathrm{MC}}$ but applied to those bytes with equal position inside the AES states. 


\section{Improved Truncated Differential Analysis of ECHO}

In this section we describe the main concepts used to attack the ECHO hash function. We first describe the improved truncated differential paths which have a very low number of active S-boxes. These sparse truncated differential paths are the core of our attacks and for a better description of the attacks, we reorder the ECHO round transformations. This reordering gives two combined building blocks of ECHO, the SuperMixColumns and SuperBox transformations. We then show how to efficiently find both differences and values through these functions for a given truncated differential path.

\subsection{Sparse Truncated Differential Paths for ECHO}

In this section we construct truncated differential paths with a low number of active bytes. Since ECHO has the same properties for words as AES has for bytes, at least 25 AES states are active in each 4-round differential path of ECHO. However, we can reduce the number of active S-boxes in each AES state to get a sparse 4-round truncated differential path with only 245 active S-boxes. Note that the truncated differential path of the previously best known analysis of ECHO has already 320 active S-boxes in a single round [18]. A trivial lower bound [1] of active S-boxes for 4 rounds is 125 .

The AES structure of ECHO ensures that the minimum number of active AES states (or words) for 4 rounds has the following sequence of active AES states:

$$
1 \stackrel{r_{1}}{\longrightarrow} 4 \stackrel{r_{2}}{\longrightarrow} 16 \stackrel{r_{3}}{\longrightarrow} 4 \stackrel{r_{4}}{\longrightarrow} 1
$$

Also, the same sequence of active bytes holds for 4 rounds of AES. In previous analysis of ECHO, truncated differential paths have been used with 16 active bytes in the AES states where the ECHO state has also 16 active words. In these attacks always one full active state with 256 active S-boxes was used. In the following, we show how to construct sparse truncated differential paths with a maximum of 64 active bytes in each single ECHO state.

The main idea is to place AES states with only one active S-box into those ECHO rounds with 16 active words. This way, the number of total active bytes (or S-boxes) can be greatly reduced. The resulting 4-round truncated differential path of ECHO is given in Fig. 1 and consists of only 245 active S-boxes. Since one round of ECHO consists of two AES rounds, it follows that the full active AES states result in those rounds of ECHO with 4 active words. The ECHO state with only one active word contains only one active byte in this AES state. Note that in the attacks on ECHO, we use this truncated differential path with small modifications to improve the overall complexity of the attacks.

\subsection{An Equivalent ECHO Round Description}

For an easier description of our attack, we use an equivalent description of one ECHO round. First, we swap the BigShiftRows transformation with the MixColumns transformation of the second AES round. Second, we swap SubBytes with ShiftRows of 
the first AES round. Swapping these operations does not change the computational result of ECHO and similar alternative descriptions have already been used in the analysis of AES. This way, we get two new super-round transformations separated just by byte shuffling operations: SuperMixColumns and SuperBox. These functions with adjacent byte shuffling operations are shown in Fig.2, In the following subsections, we describe these transformations and show how to efficiently find differences and pairs according to given truncated differential path for both transformations.

\subsection{SuperMixColumns}

The SuperMixColumns transformation combines the four MixColumns transformations of the second AES round with the 4 MixColumns transformations of BigMixColumns in the same $1 \times 16$ column slice of the ECHO state (see Fig. 2). We denote by column slice the 16 bytes of the same 1-byte wide column of the $16 \times 16$ ECHO state. Note that the BigMixColumns transformation consists of $16 \times 4$ parallel MixColumns transformations. Each of these MixColumns transformations mixes those four bytes of an ECHO column, which have the same position in the four AES states. Using the alternative description of ECHO (see Fig. 2), it is easy to see that four MixColumns operations of the second AES round work on the same column slice as four MixColumns operations of BigMixColumns. We combine these eight MixColumns transformations to get a SuperMixColumns transformation on a 1-byte wide column slice of ECHO.

We have determined the $16 \times 16$ matrix $M_{\mathrm{SMC}}$ of the SuperMixColumns transformation which is applied to the ECHO state instead of MixColumns and BigMixColumns. This matrix can be computed by the Kronecker product of two MixColumns MDS matrices $M_{\mathrm{MC}}$ and is given as follows:

$$
M_{\text {SMC }}=M_{\text {MC }} \otimes M_{\text {MC }}=\left[\begin{array}{lllll}
2 & 3 & 1 & 1 \\
1 & 2 & 3 & 1 \\
1 & 1 & 2 & 3 \\
3 & 1 & 1 & 2
\end{array}\right] \otimes\left[\begin{array}{llllll}
2 & 3 & 1 & 1 \\
1 & 2 & 3 & 1 \\
1 & 1 & 2 & 3 \\
3 & 1 & 1 & 2
\end{array}\right]=\left[\begin{array}{lllllllllllllllll}
4 & 6 & 2 & 2 & 6 & 5 & 3 & 3 & 2 & 3 & 1 & 1 & 2 & 3 & 1 & 1 \\
2 & 4 & 6 & 2 & 3 & 6 & 5 & 3 & 1 & 2 & 3 & 1 & 1 & 2 & 3 & 1 \\
2 & 2 & 4 & 6 & 3 & 3 & 6 & 5 & 1 & 1 & 2 & 3 & 1 & 1 & 2 & 3 \\
6 & 2 & 2 & 4 & 5 & 3 & 3 & 6 & 3 & 1 & 1 & 2 & 3 & 1 & 1 & 2 \\
2 & 3 & 1 & 1 & 4 & 6 & 2 & 2 & 6 & 5 & 3 & 3 & 2 & 3 & 1 & 1 \\
1 & 2 & 3 & 1 & 2 & 4 & 6 & 2 & 3 & 6 & 5 & 3 & 1 & 2 & 3 & 1 \\
1 & 1 & 2 & 3 & 2 & 2 & 4 & 6 & 3 & 3 & 6 & 5 & 1 & 1 & 2 & 3 \\
3 & 1 & 1 & 2 & 6 & 2 & 2 & 4 & 5 & 3 & 3 & 6 & 3 & 1 & 1 & 2 \\
2 & 3 & 1 & 1 & 2 & 3 & 1 & 1 & 4 & 6 & 2 & 2 & 6 & 5 & 3 & 3 \\
1 & 2 & 3 & 1 & 1 & 2 & 3 & 1 & 2 & 4 & 6 & 2 & 3 & 6 & 5 & 3 \\
1 & 1 & 2 & 3 & 1 & 1 & 2 & 3 & 2 & 2 & 4 & 6 & 3 & 3 & 6 & 5 \\
3 & 1 & 1 & 2 & 3 & 1 & 1 & 2 & 6 & 2 & 2 & 4 & 5 & 3 & 3 & 6 \\
6 & 5 & 3 & 3 & 2 & 3 & 1 & 1 & 2 & 3 & 1 & 1 & 4 & 6 & 2 & 2 \\
3 & 6 & 5 & 3 & 1 & 2 & 3 & 1 & 1 & 2 & 3 & 1 & 2 & 4 & 6 & 2 \\
3 & 3 & 6 & 5 & 1 & 1 & 2 & 3 & 1 & 1 & 2 & 3 & 2 & 2 & 4 & 6 \\
5 & 3 & 3 & 6 & 3 & 1 & 1 & 2 & 3 & 1 & 1 & 2 & 6 & 2 & 2 & 4
\end{array}\right]
$$

Note that the optimal branch number of a $16 \times 16$ matrix is 17 , which could be achieved by an MDS matrix. Using Magma we have computed the branch number of SuperMixColumns which is 8 . Hence, it is possible to find differential paths in SuperMixColumns such that the sum of active bytes at input and output is only 8. An according truncated differential path through MixColumns and BigMixColumns has the following sequence of active bytes:

$$
4 \stackrel{\mathrm{MC}}{\longrightarrow} 16 \stackrel{\mathrm{BMC}}{\longrightarrow} 4
$$




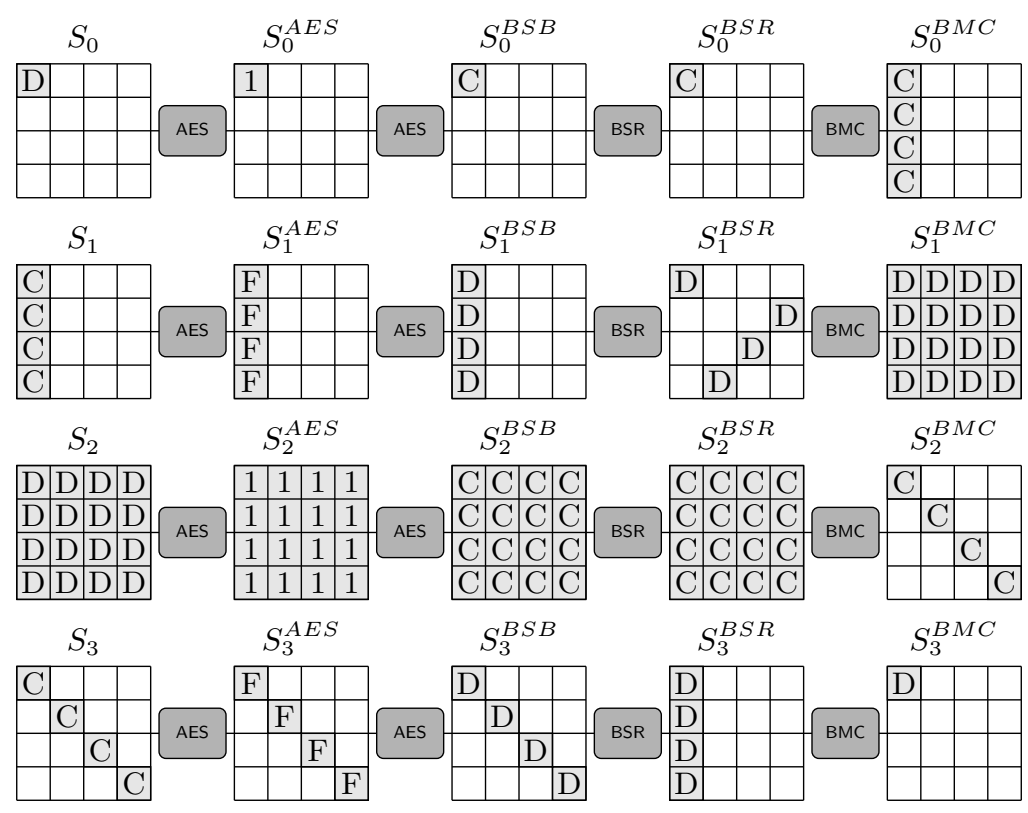

Fig. 1. The sparse truncated differential path for 4 rounds of ECHO. By 1, D, C, F we denote the pattern and number of active bytes in each AES state (also see [7]). A 1 denotes an AES state with only one active byte, a D an active diagonal (4 active bytes), a $\mathrm{C}$ an active column (4 active bytes) and an $\mathrm{F}$ denotes a full active state (16 active bytes). Note that a maximum of 64 bytes are active in each single ECHO state.

An example for a valid SuperMixColumns differential according to this truncated differential path is given as follows:

$$
\operatorname{SMC}\left([\text { E000 } 9000 \text { D000 B000 }]^{\top}\right)=[2113000000000000]^{\top}
$$

However, the probability for a truncated differential path from $4 \rightarrow 16 \rightarrow 4$ active bytes (with fixed position) through SuperMixColumns is $2^{-24}$. Hence, only $2^{8}$ (out of $2^{32}$ ) such differentials for the given position of active bytes exist. In the sparse truncated differential path of Fig. 1, this $4 \rightarrow 16 \rightarrow 4$ transition through SuperMixColumns occurs in the second and forth round.

\subsection{SuperBox}

The SuperBox has first been used by the designers of AES in the differential analysis of two AES rounds [6]. Since one round of ECHO also consists of two consecutive AES rounds we use this concept in our analysis as well. Using SuperBoxes, we can represent two rounds of AES using a single non-linear layer and two adjacent linear layers. Since we can swap the SubBytes and ShiftRows operation 

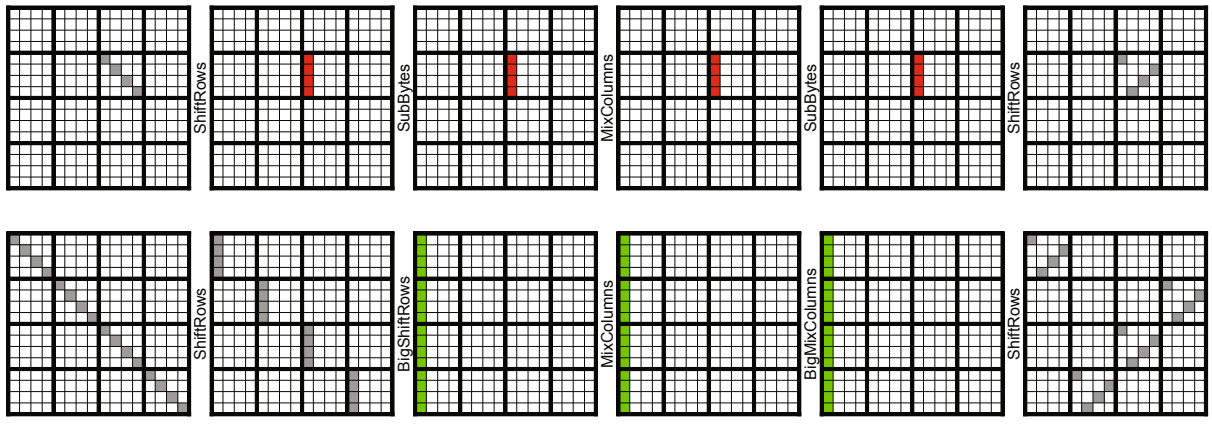

Fig. 2. The two super-round transformations of ECHO: SuperBox (top, red) and SuperMixColumns (bottom, green) with adjacent byte shuffling operations (ShiftRows and BigShiftRows)

of the first AES round, we get a sequence of SB-MC-SB transformations with independent columns in the middle. One such column is called a SuperBox and consists of 4 parallel S-boxes, one MixColumns operation and another 4 parallel S-boxes (see Fig. 2). Hence, a SuperBox is in fact a 32-bit non-linear S-box.

This separation of two AES rounds into parallel 32-bit SuperBoxes allows to efficiently find pairs for a given (truncated) differential. In a theoretical attack on ECHO or if we do not care about memory, we can simply pre-compute and store the whole differential distribution table (DDT) of the AES SuperBox with a time and memory complexity of $2^{64}$. The DDT stores which input/output differentials of the SuperBox are possible and also stores all input values such that these differentials are fulfilled. Note that in ECHO, each SuperBox is keyed in the middle by the counter value. Hence, we need different DDTs for all SuperBoxes with different keys. To reduce the memory requirements and the maximum time to find values for given SuperBox differentials, a time-memory trade-off with average complexity one and memory requirements of $2^{32}$ can be used. This method has first been proposed in the analysis of the hash function Whirlpool [9, Appendix A] and applied to Grøstl in [15]. The same technique has been discovered independently in [7].

\subsection{Expected Number of Pairs}

At this point, we can already compute the expected number of pairs conforming to the 4-round truncated differential path given in Fig. 1, The resulting number of solutions determines the degrees of freedom we have in the attack. At the input of the path, we have a 2048-bit value and differences in 4 bytes. Therefore, the total number of possible inputs pairs (excluding the 128-bit salt) is about

$$
2^{2048} \cdot 2^{8 \cdot 4}=2^{8 \cdot 260}=2^{2080} .
$$

In general, the probability for a random pair to follow a truncated differential path from $a$ to $b$ active bytes (with $a+b \geq 5$ ) through MixColumns is $2^{-8 \cdot(4-b)}$. An 
exception is the propagation from $4 \rightarrow 16 \rightarrow 4$ bytes through SuperMixColumns, which has a probability of $2^{-24}$ (see Sect. 3.3). Multiplying all probabilities through MixColumns and SuperMixColumns gives the approximate probability for a random input pair to follow the whole truncate differential path. For the path given in Fig. 1, we get a probability significantly less than one for all MixColumns or SuperMixColumns transformation where a reduction in the number of active bytes occur. This happens in the 1 st $M C$ of round $1(D-1)$, the 2 nd $M C$ of round $2(4 \times \mathrm{F}-\mathrm{D})$, the 1 st MC $(16 \times \mathrm{D}-1)$ and SMC $(4 \times 1111-\mathrm{FFFF}-$ F000) of round 3 , and the 2 nd MC $(4 \times \mathrm{F}-\mathrm{D})$ and BMC $(3 \times \mathrm{D}-0)$ of round 4 . We then get for the total probability of the truncated differential path (in base 2 logarithm):

$$
-8 \cdot(3+4 \cdot 12+16 \cdot 3+4 \cdot 3+4 \cdot 12+3 \cdot 4)=-8 \cdot 171
$$

So in total, the expected number of solutions for this path is

$$
2^{8 \cdot 260} \cdot 2^{-8 \cdot 171}=2^{8 \cdot 89}=2^{712}
$$

and we have about 712 degrees of freedom in this 4-round truncated differential path.

\section{Attacks on the ECHO-256 Hash Function}

In this section we use the sparse truncated differential path and properties of SuperMixColumns to get attacks for up to 5 rounds of the ECHO-256 hash function. We first describe our main result, the subspace distinguisher for 5 rounds of ECHO256 in detail. Then, we briefly show how to get near-collisions for 4.5 rounds and collisions for 4 rounds of ECHO-256.

\subsection{Subspace Distinguisher for 5 Rounds}

In this section we show that ECHO-256 reduced to 5 rounds can be distinguished from an ideal hash function. We are able to construct a large set of output differences which fall into a vector space of fixed dimension. But when does this result in a distinguisher on the hash function? An attacker could have chosen the vector space specifically to fit a previously computed set of differences. Also, finding up to $x$ differences in subspace of dimension $x$ is trivial, even for ideal functions. But once a subspace has been chosen, finding additional differences in this subspace should again have the generic complexity. We have a similar situation for preimage attacks: finding a preimage is trivial if the attacker can choose the hash value. Note that in most distinguishing attacks, the generic complexity also depends on the number of found solutions. To compare distinguishers with generic attacks, differential $q$-multicollisions have been used in the distinguishing attacks on AES [3]. More general, to analyze the complexity of finding differences in a vector space of fixed dimension, the subspace distinguisher has 
been introduced in the analysis of Whirlpool [9, 10. Before we describe the subspace distinguisher for 5 rounds of ECHO-256 in detail, we give an overview of the truncated differential path and provide a brief outline of the attack.

The Truncated Differential Path. For the attack we use two message blocks where the first block does not contain differences. For the second message block, we use the truncated differential path given in Fig. 3. We use colors (red, yellow, green, blue, cyan) to describe different phases of the attack and to denote their resulting solutions. Active bytes are denoted by black color and all AES states are active which contain at least one active byte. Hence, the sequence of active AES states for each round of ECHO is as follows:

$$
5 \stackrel{r_{1}}{\longrightarrow} 16 \stackrel{r_{2}}{\longrightarrow} 4 \stackrel{r_{3}}{\longrightarrow} 1 \stackrel{r_{4}}{\longrightarrow} 4 \stackrel{r_{5}}{\longrightarrow} 16
$$

Note that in this path we keep the number of active bytes low as described in Sect. 3.1. Except for the beginning and end, at most one fourth of the ECHO state is active and therefore, we have enough freedom to find many solutions. Since the lower half of the state is truncated, we have most differences in the lower half of the message and there are no differences in the chaining input (blue). The padding of the second (and last) message block is denoted by cyan bytes. The last 16 bytes (one AES state) of the padding contain the message length, and the two bytes above contain the 2-byte value with the hash size. Note that the AES states containing the chaining values (blue) and padding (cyan) do not get mixed with other AES states until the first BigMixColumns transformation.

Attack Outline. To find input pairs according to this path we use the rebound attack [14] with multiple inbound phases [9,12. The main advantage of multiple inbound phases is that we can first find pairs for each inbound phase independently and then, connect (or merge) the results. For the attack on 5 rounds of ECHO-256 we use an inbound phase in round 2 (red) and another inbound phase in round 3 (yellow). The 1st inbound phase finds values and differences for the red bytes which we connect with the chaining input (blue) and padding (cyan) by merging lists. Then, we compute the solutions of the 2 nd inbound phase forwards in the outbound phase (green) to insure the propagation according to the truncated differential path until the end. Finally, we merge the solutions of the two inbound phases by determining the remaining (white) values using a generalized birthday attack on 4 independent columns of the state. Note that in some cases, the probability to find one solution is only close to one. However, for simplicity reasons of describing the attack we assume it is one, since we have enough freedom in the attack to repeat all phases with different starting points to get one solution on average.

1st Inbound. We start the 1st inbound phase with a random difference according to the truncated differential path through SuperMixColumns between state $S_{14}$ and state $S_{16}$ (see Sect.3.3). We compute these differences backward to get the output differences of the SuperBoxes in state $S_{12}$. For each column in state $S_{7}$ we choose $2^{32}$ random differences for the given active bytes. We compute these differences forward through BigMixColumns to the input of the SuperBoxes. Note that for the 

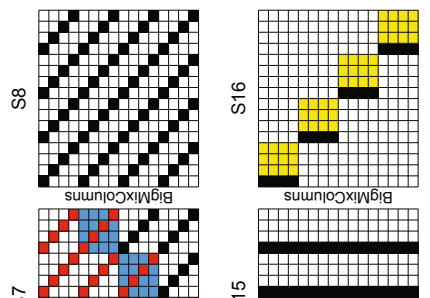

ஸे

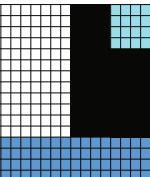

SMOYH!Ys
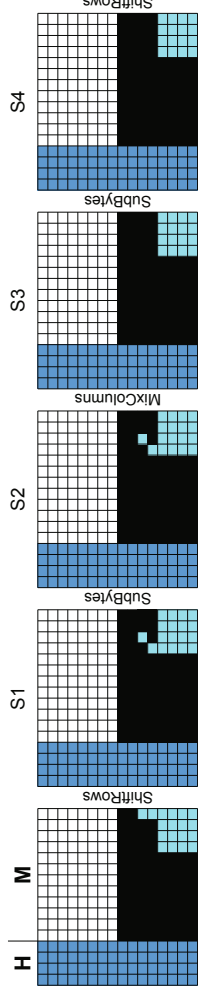

๙ั


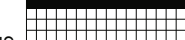
के वाणता ๓\#\#+\# sumn|0JX!W \begin{tabular}{|l|l|l|l|l|}
\hline \hline & & & & \\
\hline
\end{tabular} 六 ב

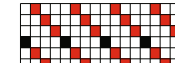

m

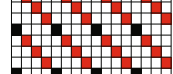
Hח
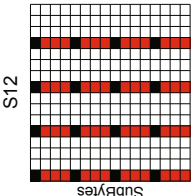
səłkgqns

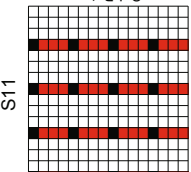

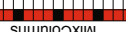
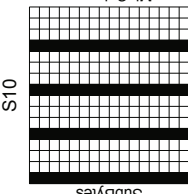

ПШШППППП
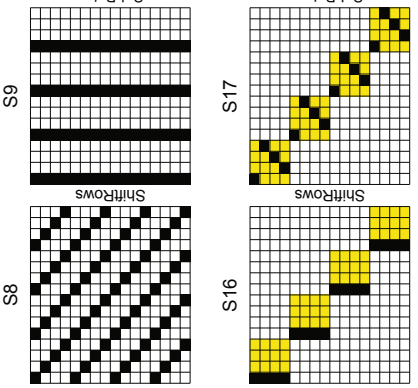

sumn|0JX!W

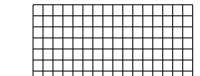


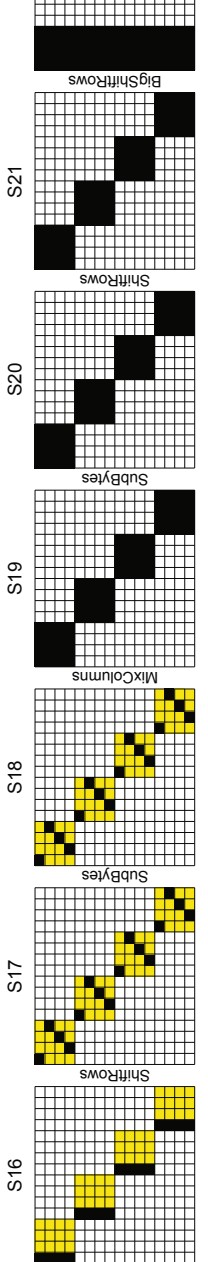


สี

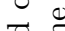

刍

ฮ..

$\stackrel{5}{5}$

s.

$>$

产。

$0>$
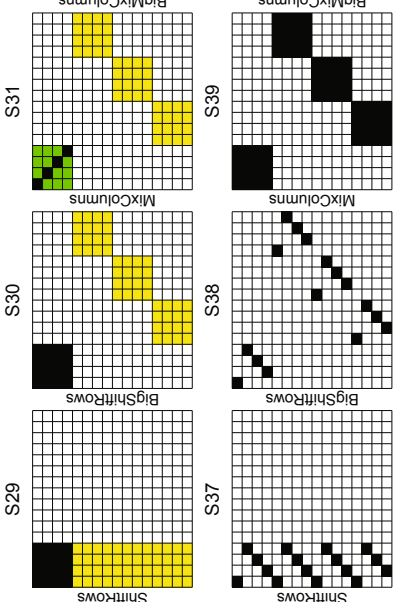

๙ิ

$\widehat{\bar{D}}$

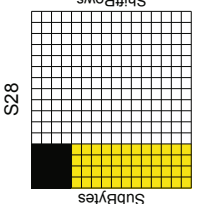

ธิ
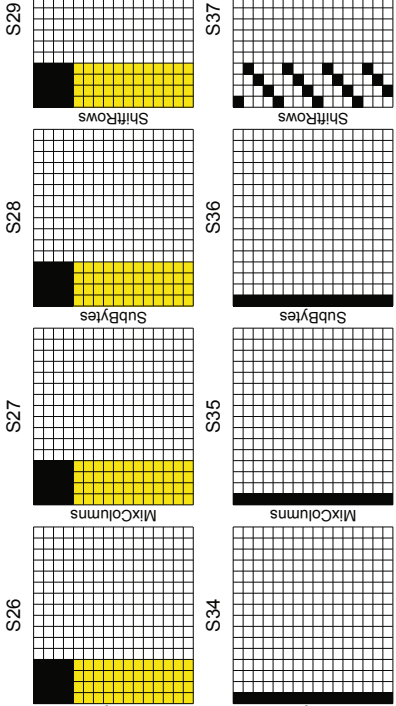

səłKgans

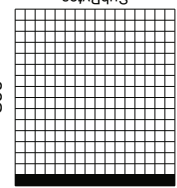

sumn!ojx!n

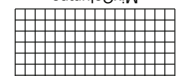

芯
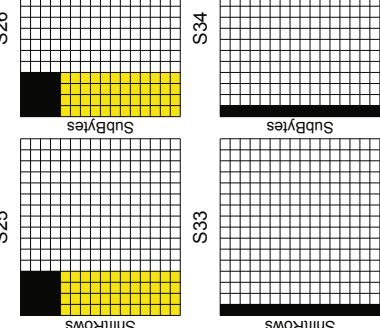

sə)रaqn

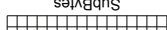

Пथम

沉
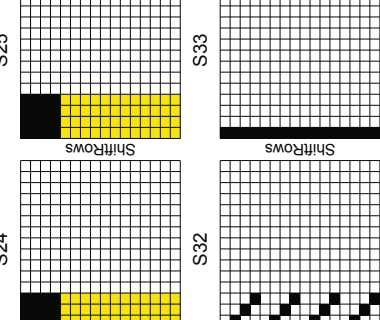

SMOYH!4

สี ญे

कै

?

물

일

口..

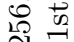

웡

要

되.尹

पै

욜

$\exists$

잉

10

运

武

$\exists$

500

害

금

0

.

on $\Xi$

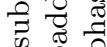

$\approx 2$

리

$+8$

$\stackrel{\circ}{\ddagger}$

표.

2.

.]త్.$\Xi$

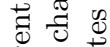

屯ै है

$\because>$.

응

ॠ

클

¿

웜

E 0

ค่ สี

घै

$\therefore$ 
last column we could choose up to $2^{64}$ differences ( 8 active bytes), whereas in all other columns we have only $2^{32}$ possible differences ( 4 active bytes).

As described in Sect. 3.4. we find values according to the SuperBox differentials with an average complexity of 1 by using DDT lookups. Note that for some differentials no solutions exist, but for each possible differential we get more pairs which out-weight the non-existing ones (for more details we refer to [14]). In general, for each active AES S-box a differential is possible with a probability of about $2^{-1}$ and we get at least 2 pairs. Hence, for a full active AES state, one out of approximately $2^{16}$ differences gives a differential match and then, provides at least $2^{16}$ solutions. In the following attacks, it is reasonable to assume that for each differential we get one solution with average complexity one.

Since in the 1st inbound phase the columns of ECHO are independent, we get $2^{32}$ independent solutions for each of the four columns in state $S_{7}$ (red and black bytes) with complexity $2^{32}$ in time and memory. These solutions (or pairs) consist of differences and values for the black bytes, and values for the red bytes in $S_{7}$. Note that for each solution (and arbitrary choice of white bytes in $S_{7}$ ) the truncated differential path from state $S_{3}$ to state $S_{23}$ is already fulfilled.

Merge Chaining Input. Next, we need to merge the solutions of the 1st inbound phase with the chaining input and bytes fixed by the padding. Therefore, we choose $2^{32}$ random first message blocks and compute the resulting chaining value after one compression function call of ECHO. Note that each AES state can be independently computed forward to state $S_{7}$ until the first BigMixColumns transformation. We do this for the chaining values (blue) and the AES state containing the message length (cyan). Note that we match the two remaining bytes and one bit of the padding at a later step.

We merge the $2^{32}$ chaining values with the solutions of the 1 st inbound phase column by column. We start with column 0 where we need to match the padding state as well. Since we match 64 bits of overlapping red and blue/cyan bytes, the expected number of solutions is $2^{32} \times 2^{32} \times 2^{-64}=1$. We compute this solution by merging the two lists of size $2^{32}$ and exploiting the birthday effect. For all other columns, we need to match only 4 red bytes in each blue AES state and we get $2^{32} \times 2^{-32}=1$ solution as well. Since we only merge lists of size $2^{32}$ the complexity of this step is $2^{32}$ in time and memory.

After this step, we have found solutions where the values of all blue, cyan and red bytes, as well as the values of the black bytes between state $S_{7}$ and state $S_{14}$ are determined. Furthermore, all differences (black bytes) from state $S_{4}$ up to state $S_{17}$ can be computed.

2nd Inbound. In the 2nd inbound phase, we search for values and differences such that the truncated differential path in round 3 is fulfilled (yellow). Remember that the differences in state $S_{17}$ have already been fixed due to the 1 st inbound phase. We start with $2^{64}$ differences of state $S_{24}$ and compute backwards to state $S_{20}$, the output of the SuperBoxes. Note that we have 16 independent SuperBoxes for the yellow AES states between state $S_{17}$ and $S_{20}$. Again, we use the DDT of the SuperBoxes to find the according values for the given differentials. For 4 full 
active AES states, the probability of a differential is about $2^{-4 \cdot 16}$. Among the $2^{64}$ differentials, we expect to find one possible differential. Note that for a valid differential, the expected number of solutions for the 2 nd inbound phase is $2^{64}$. For each of these pairs, differences and values of all yellow and black bytes in round 3 are determined.

Outbound Phase. Next, we compute the $2^{64}$ differences and values of state $S_{24}$ forward to $S_{31}$. With a probability of $2^{-96}$ we get 4 active bytes after MixColumns in state $S_{31}$. Hence, we have to repeat the 2 nd inbound phase $2^{32}$ times to find one solution for the outbound phase as well and we get a total complexity of $2^{96}$. After this step, the complete truncated differential path is fulfilled (except for two cyan bytes in the first states). Furthermore, all differences (black bytes) from state $S_{4}$ until state $S_{33}$ are already determined. Also, the values of the yellow, red, blue, green and cyan bytes, and the values of the black bytes from state $S_{7}$ to $S_{31}$ except for state $S_{15}$ are determined. What remains is to find values for the white bytes such that the results of the two inbound phases (blue/cyan/red and yellow bytes) can be connected.

Merge Inbound. To merge the two inbound phases, we need to find according values for the white bytes. We first choose random values for all remaining bytes of the first two columns in state $S_{7}$ (gray and lightgray) and compute them forward to state $S_{14}$. Note that we need to try $2^{2 \cdot 8+1}$ values for AES state $S_{7}[2,1]$ to also match the 2-byte (cyan) and 1-bit padding at the input in AES state $S_{0}[2,3]$. To illustrate all further steps, we use only states and colors shown in Fig. 4. Note that all gray, lightgray and brows bytes have already been determined either by an inbound phase, chaining value, padding or just by choosing random values for the remaining free bytes of the first two columns of $S_{7}$. Also the cyan bytes are fixed already. However, all white, red, green, yellow and blue bytes are still free to choose.

By taking a look at the linear SuperMixColumns transformation, we observe that in each column slice, 14 out of 32 input/output values are already fixed. Hence, we expect to get $2^{16}$ solutions for this linear system of equations. Unfortunately, for the given position of already determined 14 bytes, the linear system of equations does not have a full rank. One can determine the resulting
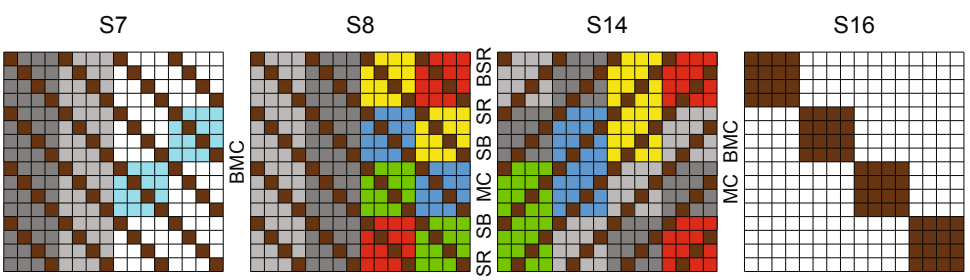

Fig. 4. States used to merge the two inbound phases with the chaining values. Gray, lightgray and brown bytes show values already determined. Green, blue, yellow and red bytes show independent values used in the generalized birthday attack and cyan bytes represent values with the target conditions. 
system using the matrix $M_{\mathrm{SMC}}$ of SuperMixColumns. For the first column-slice the system is given as follows:

$$
\begin{gathered}
M_{\mathrm{SMC}} \cdot\left[a_{0} L_{0} L_{1} L_{2} a_{1} L_{0}^{\prime} L_{1}^{\prime} L_{2}^{\prime} a_{2} x_{0} x_{1} x_{2} a_{3} x_{3} x_{4} x_{5}\right]^{T}= \\
{\left[b_{0} b_{1} b_{2} b_{3} y_{0} y_{1} y_{2} y_{3} y_{4} y_{5} y_{6} y_{7} y_{8} y_{9} y_{10} y_{11}\right]}
\end{gathered}
$$

The free variables in this system are $x_{0}, \ldots, x_{5}$ (green). The values $a_{0}, a_{1}, a_{2}, a_{3}$, $b_{0}, b_{1}, b_{2}, b_{3}$ (brown) have been determined by the first or second inbound phase, and the values $L_{0}, L_{1}, L_{2}$ (lightgray) and $L_{0}^{\prime}, L_{1}^{\prime}, L_{2}^{\prime}$ (gray) are determined by the choice of arbitrary values in state $S_{7}$. Since the values $y_{0}, \ldots, y_{11}$ (white) are free to choose we can remove their respective equations. We move terms which do not depend on $x_{i}$ to the right side and get the following linear system with 4 equations and 6 variables:

$$
\left[\begin{array}{llllll}
3 & 1 & 1 & 3 & 1 & 1 \\
2 & 3 & 1 & 2 & 3 & 1 \\
1 & 2 & 3 & 1 & 2 & 3 \\
1 & 1 & 2 & 1 & 1 & 2
\end{array}\right] \cdot\left[\begin{array}{l}
x_{0} \\
x_{1} \\
x_{2} \\
x_{3} \\
x_{4} \\
x_{5}
\end{array}\right]=\left[\begin{array}{l}
c_{0} \\
c_{1} \\
c_{2} \\
c_{3}
\end{array}\right]
$$

On the right side, we have the constant values $c_{0}, c_{1}, c_{2}, c_{3}$ which are determined by $a_{0}, a_{1}, a_{2}, a_{3}, b_{0}, b_{1}, b_{2}, b_{3}, L_{0}, L_{1}, L_{2}, L_{0}^{\prime}, L_{1}^{\prime}, L_{2}^{\prime}$ and we get for example:

$$
c_{0}=b_{0}+4 a_{0}+6 L_{0}+2 L_{1}+2 L_{2}+6 a_{1}+5 L_{0}^{\prime}+3 L_{1}^{\prime}+3 L_{2}^{\prime}+2 a_{0}+2 a_{1}
$$

The matrix of this linear system has rank 3 instead of 4 and therefore, we only get a solution with probability $2^{-8}$. We can solve this system of equations by transforming the system into echelon form and get:

$$
\left[\begin{array}{llllll}
1 & 0 & 0 & 1 & 0 & 0 \\
0 & 1 & 0 & 0 & 1 & 0 \\
0 & 0 & 1 & 0 & 0 & 1 \\
0 & 0 & 0 & 0 & 0 & 0
\end{array}\right] \cdot\left[\begin{array}{l}
x_{0} \\
x_{1} \\
x_{2} \\
x_{3} \\
x_{4} \\
x_{5}
\end{array}\right]=\left[\begin{array}{l}
c_{0}^{\prime} \\
c_{1}^{\prime} \\
c_{2}^{\prime} \\
c_{3}^{\prime}
\end{array}\right]
$$

where the values $c_{0}^{\prime}, c_{1}^{\prime}, c_{2}^{\prime}, c_{3}^{\prime}$ are a linear combination of $c_{0}, c_{1}, c_{2}, c_{3}$. From the last equation, we get the 8 -bit condition $c_{3}^{\prime}=0$. Since also $c_{3}^{\prime}$ depends linearly on $L_{i}$ and $L_{i}^{\prime}$, we can separate this linear equation into terms depending only on values of $L_{i}$ and only on $L_{i}^{\prime}$, and get $c_{3}^{\prime}=f_{1}\left(L_{i}\right)+f_{2}\left(L_{i}^{\prime}\right)+f_{3}\left(a_{i}, b_{i}\right)=0$. For all other 16 column-slices and fixed positions of gray bytes, we also get matrices of rank 3 . In total, we get 16 8-bit conditions and the probability to find a solution for a given choice of gray and lightgray values in state $S_{14}$ and $S_{16}$ is $2^{-128}$. However, we can also find a solution using the birthday effect with a complexity of $2^{64}$ in time and memory.

First, we start by choosing $2^{64}$ values for each of the first (gray) and second (lightgray) BigColumn in state $S_{7}$. We compute these values independently forward to state $S_{14}$ and store them in two lists $L$ and $L^{\prime}$. We also separate all equations of the 128-bit condition into parts depending only on values of $L$ and $L^{\prime}$. We apply the resulting functions $f_{1}, f_{2}, f_{3}$ to the elements of lists $L_{i}$ and $L_{i}^{\prime}$, 
and search for matches between the two lists using the birthday effect. Now, by solving (3) we get $2^{24}$ solutions for the fist column-slice. By doing the same for all other slices, we get $2^{24}$ independent solutions for each column-slice. Hence, in total we can get up to $2^{16 \cdot 24}=2^{384}$ solutions for the whole ECHO state.

We continue with a generalized birthday match to find values for all remaining bytes of the state. For each column in state $S_{14}$, we independently choose $2^{64}$ values for the green, blue, yellow and red columns, and compute them independently backward to $S_{8}$. We need to match the values of the cyan bytes of state $S_{7}$, which results in a condition on 24 bytes or 192 bits. Since we have 4 independent lists with $2^{64}$ values in state $S_{8}$, we can use the generalized birthday attack [20] to find one solution with a complexity of $2^{192 / 3}=2^{64}$ in time and memory. In detail, we need to match values after the BigMixColumns transformation in backward direction. Hence, we first multiply each byte of the 4 independent lists by the 4 multipliers of the InvMixColumns transformation. Then, we get 24 equations containing only XOR conditions on bytes between the target value and elements of the 4 independent lists. This can be solved using a generalized birthday attack.

After this step, all values and differences are determined. We can compute the input message pair, as well as the output differences for ECHO-256 reduced to 5 rounds. By simply repeating the merge inbound phase $2^{32}$ times, we can find at least $2^{32}$ solutions for the whole truncated differential path. The total complexity is still $2^{96}$ compression function evaluations and memory requirements of $2^{64}$.

Subspace Distinguisher. Note that one message pair resulting in one output differences does not give a distinguisher. We need to find many output differences in a subspace with a complexity less than in the generic case. To determine the generic complexity of finding output differences in a vector space and the resulting advantage of our attack we use the subspace distinguisher. In general, the size of the output vector space is define by the number of active bytes prior to the linear transformations in the last round (16 active bytes after the last SubBytes), combined with the number of active bytes at the input due to the feed-forward ( 0 active bytes in our case). This would results in a vector space dimension of $(16+0) \cdot 8=128$. However, a weakness in the combined transformations SuperMixColumns, BigFinal and output truncation reduces the vector space to a dimension of 64 at the output of the hash function (for the given truncated differential path).

Note that we can move the BigFinal function prior to SuperMixColumns, since BigFinal is a linear transformation and the same linear transformation $M_{\mathrm{SMC}}$ is applied to all columns in SuperMixColumns. Hence, we get 4 active bytes in each column slice at the same position in each AES state. To each (active) column slice $C_{16}$, we first apply the SuperMixColumns multiplication with $M_{\mathrm{SMC}}$ and then, a matrix multiplication with $M_{\text {trunc }}$ which truncates the lower 8 rows. Since only 4 bytes are active in $C_{16}$, these transformations can be combined into a transformation using a reduced $4 \times 8$ matrix $M_{\text {comb }}$ applied to the reduce input $C_{4}$, which contains only the 4 active bytes of $C_{16}$ :

$$
M_{\text {trunc }} \cdot M_{\mathrm{SMC}} \cdot C_{16}=M_{\text {comb }} \cdot C_{4}
$$


The multiplication with zero differences of $C_{16}$ removes 12 columns of $M_{\mathrm{SMC}}$ while the truncation removes 8 rows of $M_{\mathrm{SMC}}$. An example for the first active column slice is given as follows:
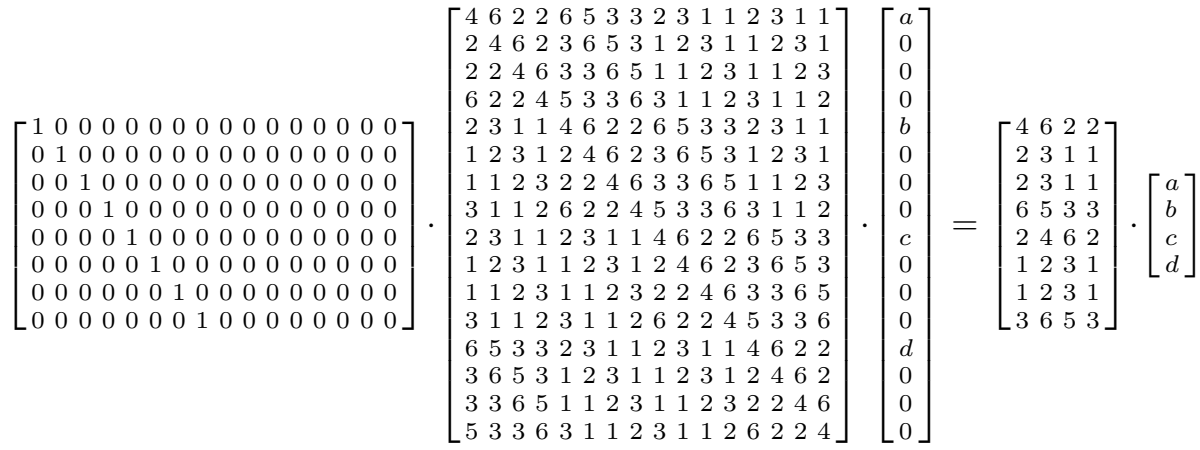

Analyzing the resulting matrix $M_{\text {comb }}$ for all 4 active column slices shows that in each case, the rank of $M_{c o m b}$ is 2 instead of 4 . This reduces the dimension of the vector space in each active column slice from 32 to 16 . Since we have 4 active columns, the total dimension of the vector space at the output of the hash function is 64 .

We use [9, Corollary 1] and [9, Equation (19)] to compute the complexity of a generic distinguishing attack on the ECHO-256 hash function. We get the parameters $N=256$ (hash function output size), $n=64$ (dimension of vector space) and $t=2^{32}$ (number of outputs in vector space) for the subspace distinguisher. Then, the generic complexity to construct $2^{32}$ elements in a vector space of dimension 64 is about $2^{111.8}$ compression function evaluations. Remember that in our attack on ECHO we also get $2^{32}$ pairs in a vector space of the same dimension. Hence, the total complexity for our subspace distinguisher on 5 rounds of the ECHO-256 hash function is about $2^{96}$ compression function evaluations with memory requirements of $2^{64}$.

\subsection{Collisions for 4 Rounds}

Finally, we are able to construct collisions for 4 rounds of the ECHO-256 hash function. The attack and truncated differential path is similar as for the subspace distinguisher on 5 rounds. We use a two-block message and the truncated differential path for the second block is given in Fig. 5. Again, we start with the 1st inbound phase, merge the chaining input and continue with the 2 nd inbound and outbound phase. To get a collision at the output we use differences in the feed-forward and do a 3rd inbound phase in two AES states in round 1. Finally, we merge the solutions of the two inbound phases to determining the remaining values. In the following, we only describe parts of the attack which are new or have been changed.

1st Inbound. The 1st inbound phase is the same as for the subspace distinguisher, except that in state $S_{7}$ we choose $2^{64}$ random differences for the active bytes of column 0 and 1 , and $2^{32}$ random differences for column 2 and 3 . Hence, after the 1 st 

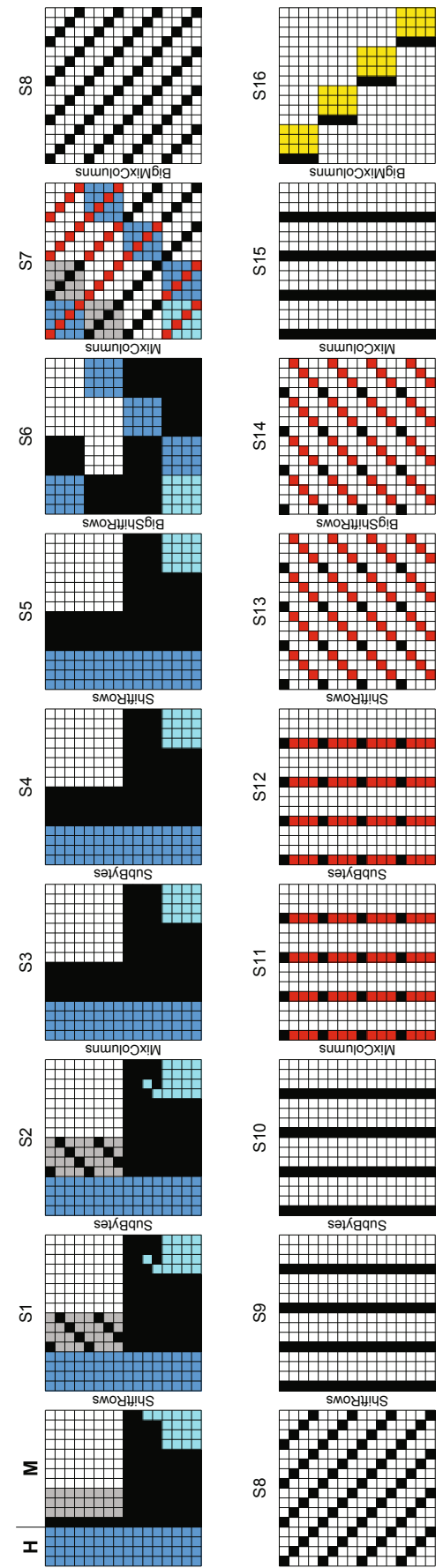
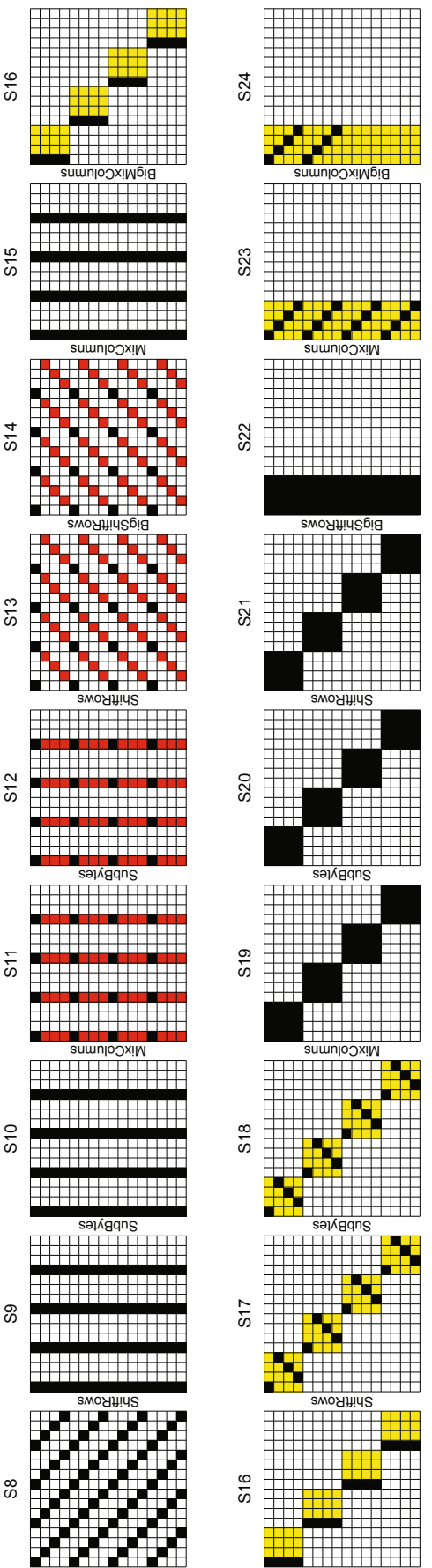

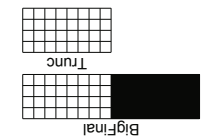

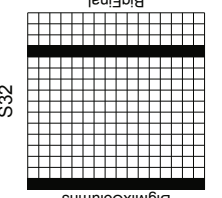

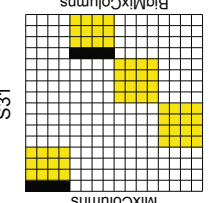

ర్ల

घี

훙

艺

สี . .

品

สี

उे.

手

$\stackrel{0}{0}$

궁

c을

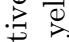

范

๘ี

ส 0

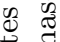

2

둘

ส

$\overrightarrow{0}$

0.7

No

연 른

望

फै.

की गृ

$\stackrel{\infty}{\mathscr{\infty}}$

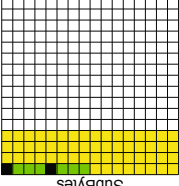

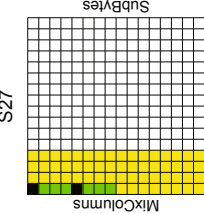

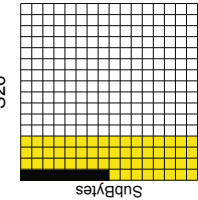

ஸू

丹

Q.

E 过

10

ฌ્ّ

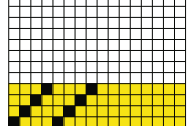

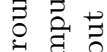

ㅇํㅇ.

oี 0

곯

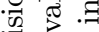

○

क 3

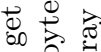

0 응

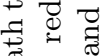

2.

丞司

ల

車已

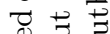

\pm 의

ॠ

글

$\Xi 50$

ن.

任合莂 
inbound phase we get $2^{64}$ independent solutions for each of the first two columns, and $2^{32}$ solutions for each of the last two columns in state $S_{7}$ (red and black bytes).

Merge Chaining Input. Again, we choose $2^{32}$ random first message blocks and merge them with the solutions of the 1 st inbound phase column by column. We start with column 0 where we need to match the padding state as well. Since we match 64 bits of overlapping red and blue/cyan bytes, the expected number of solutions is $2^{32} \times 2^{64} \times 2^{-64}=2^{32}$. For all other columns, we need to match only the 4 red bytes in each blue AES state with a probability of $2^{-32}$. For column 1 we get $2^{32}$ solutions since we have computed $2^{64}$ results in the 1 st inbound phase. For column 2 and 3 , we have $2^{32}$ solutions from the 1 st inbound phase and get one match on the overlapping 4 bytes.

2nd Inbound. To get the first solution for the 2 nd inbound phase we need to try $2^{64}$ differences of state $S_{24}$, since the probability of a differential match in 4 full active AES states is only about $2^{-4 \cdot 16}$. Then, the expected number of solutions for the 2 nd inbound phase is $2^{64}$ but we only need $2^{48}$ solutions to continue.

Outbound Phase. In the outbound phase we compute these $2^{48}$ differences and values of state $S_{24}$ forward to $S_{27}$. With a probability of $2^{-48}$ we get one active byte after MixColumns in each active state of $S_{27}$. After this step, the complete truncated differential path (except for the three first states) is fulfilled. What remains is to determine differences in the first state to get a collision at the output and to find values for the white bytes.

3rd Inbound. To get a collision at the output, we use two additional active AES states in round 1 . In $S_{0}[0,1]$ and $S_{0}[1,1]$, only the first column should be active such that the active bytes overlap with the active bytes at the output. For these active bytes at the input, we choose the differences to be $S_{0}[0,1]=S_{32}[0,0] \oplus$ $S_{32}[0,3]$ and $S_{0}[1,1]=S_{32}[1,0] \oplus S_{32}[1,3]$. Then, these differences cancel each other by the feed-forward and we get a collision. In a 3rd inbound phase, we determine the remaining values of the gray and black bytes such that the given truncated differential for these two AES states in round 1 is satisfied. Again, we can find such values and differences with a complexity of about 1 using the DDT of the SuperBoxes, and compute $2^{32}$ solutions for each AES state. Since we still have $2^{32}$ solutions for each of column 0 and column 1 due to the 1 st inbound phase, we expect to find a match for both differences and values of the overlapping 4 diagonal bytes of AES state $S_{7}[0,1]$ and $S_{7}[1,0]$.

Merge Inbound. Finally, we merge the 1st and 2nd inbound phases as in the previous attacks. Then, all values and differences are determined and we can compute the input message pair which results in a collision for ECHO-256 reduced to 4 rounds. The total complexity is $2^{64}$ in time and memory.

\section{Conclusion}

In this work we have presented the first analysis of the ECHO hash function. We give a subspace distinguisher for 5 rounds and collisions for 4 out of 8 rounds of 
the ECHO-256 hash function. Our results improve upon the previous results which are only on the (double-pipe) compression function of ECHO and for less rounds. Note that also near-collision resistance is a NIST requirement for a future SHA3 [17. In the extended version of this work [19] we provide near-collisions for 4.5/8 rounds of the hash function. Additionally we show distinguishers for 7 rounds and near-collisions for up to 6.5 rounds of the ECHO-256 and ECHO-512 without chosen salt.

In our improved attacks we combine the MixColumns transformation of the second AES round with the subsequent BigMixColumns transformation to a combined SuperMixColumns transformation. This allows us to construct very sparse truncated differential paths. In these paths, at most one fourth of the bytes are active throughout the whole computation of ECHO. This behavior is not known from the AES or AES based hash functions which strictly follow the wide-trail design strategy. Additionally, we are able to apply a rebound attack with multiple inbound phases to ECHO by using a generalized birthday technique to merge the inbound phases. Future work includes the search for even sparser truncated differential paths and the improvement of the given attacks by using the large degrees of available freedom. Also the separate search for differences and values as proposed in [13] and [8] may be used to improve the complexity of additional inbound phases.

\section{Acknowledgements}

We thank the members of the IAIK Krypto group, the designers of ECHO and especially Jérémy Jean and Florian Mendel for their comments and useful discussions. This work was supported in part by the Austrian Science Fund (FWF), project P21936, by the European Commission through the ICT programme under contract ICT-2007-216676 ECRYPT II, and by the IAP Programme P6/26 BCRYPT of the Belgian State (Belgian Science Policy).

\section{References}

1. Benadjila, R., Billet, O., Gilbert, H., Macario-Rat, G., Peyrin, T., Robshaw, M., Seurin, Y.: SHA-3 Proposal: ECHO. Submission to NIST (2008), http://crypto.rd.francetelecom.com/echo

2. Biham, E., Dunkelman, O.: A Framework for Iterative Hash Functions - HAIFA. Cryptology ePrint Archive, Report 2007/278 (2007), http://eprint.iacr.org/2007/278

3. Biryukov, A., Khovratovich, D., Nikolić, I.: Distinguisher and Related-Key Attack on the Full AES-256. In: Halevi, S. (ed.) CRYPTO 2009. LNCS, vol. 5677, pp. 231249. Springer, Heidelberg (2009)

4. Daemen, J., Rijmen, V.: The Wide Trail Design Strategy. In: Honary, B. (ed.) Cryptography and Coding 2001. LNCS, vol. 2260, pp. 222-238. Springer, Heidelberg (2001)

5. Daemen, J., Rijmen, V.: The Design of Rijndael: AES - The Advanced Encryption Standard. Springer, Heidelberg (2002) 
6. Daemen, J., Rijmen, V.: Understanding Two-Round Differentials in AES. In: De Prisco, R., Yung, M. (eds.) SCN 2006. LNCS, vol. 4116, pp. 78-94. Springer, Heidelberg (2006)

7. Gilbert, H., Peyrin, T.: Super-Sbox Cryptanalysis: Improved Attacks for AES-Like Permutations. In: Hong, S., Iwata, T. (eds.) FSE 2010. LNCS, vol. 6147, pp. 365383. Springer, Heidelberg (2010)

8. Khovratovich, D., Naya-Plasencia, M., Röck, A., Schläffer, M.: Cryptanalysis of Luffa v2 Components. In: Biryukov, A., Gong, G., Stinson, D.R. (eds.) SAC 2010. LNCS, vol. 6544. Springer, Heidelberg (2011)

9. Lamberger, M., Mendel, F., Rechberger, C., Rijmen, V., Schläffer, M.: Rebound Distinguishers: Results on the Full Whirlpool Compression Function. In: Matsui [11], pp. 126-143

10. Lamberger, M., Mendel, F., Rechberger, C., Rijmen, V., Schläffer, M.: The Rebound Attack and Subspace Distinguishers: Application to Whirlpool. Cryptology ePrint Archive, Report 2010/198 (2010), http://eprint.iacr.org/2010/198

11. Matsui, M. (ed.): ASIACRYPT 2009. LNCS, vol. 5912. Springer, Heidelberg (2009)

12. Matusiewicz, K., Naya-Plasencia, M., Nikolic, I., Sasaki, Y., Schläffer, M.: Rebound Attack on the Full Lane Compression Function. In: Matsui [11], pp. 106-125

13. Mendel, F., Peyrin, T., Rechberger, C., Schläffer, M.: Improved Cryptanalysis of the Reduced Grøstl Compression Function, ECHO Permutation and AES Block Cipher. In: Jacobson Jr., M.J., Rijmen, V., Safavi-Naini, R. (eds.) SAC 2009. LNCS, vol. 5867, pp. 16-35. Springer, Heidelberg (2009)

14. Mendel, F., Rechberger, C., Schläffer, M., Thomsen, S.S.: The Rebound Attack: Cryptanalysis of Reduced Whirlpool and Grøstl. In: Dunkelman, O. (ed.) FSE 2009. LNCS, vol. 5665, pp. 260-276. Springer, Heidelberg (2009)

15. Mendel, F., Rechberger, C., Schläffer, M., Thomsen, S.S.: Rebound Attacks on the Reduced Grøstl Hash Function. In: Pieprzyk, J. (ed.) CT-RSA 2010. LNCS, vol. 5985, pp. 350-365. Springer, Heidelberg (2010)

16. National Institute of Standards and Technology (NIST). FIPS PUB 197: Advanced Encryption Standard. Federal Information Processing Standards Publication 197, U.S. Department of Commerce (November 2001), http://www.itl.nist.gov/fipspubs

17. National Institute of Standards and Technology (NIST). Announcing Request for Candidate Algorithm Nominations for a New Cryptographic Hash Algorithm (SHA-3) Family. Federal Register 27(212), 62212-62220 (November 2007), http://csrc.nist.gov/groups/ST/hash/documents/FR_Notice_Nov07.pdf

18. Peyrin, T.: Improved Differential Attacks for ECHO and Grøstl. Cryptology ePrint Archive, Report 2010/223 (2010), http://eprint.iacr.org/2010/223

19. Schläffer, M.: Subspace Distinguisher for 5/8 Rounds of the ECHO-256 Hash Function. Cryptology ePrint Archive, Report 2010/321 (2010), http://eprint.iacr.org/2010/321

20. Wagner, D.: A Generalized Birthday Problem. In: Yung, M. (ed.) CRYPTO 2002. LNCS, vol. 2442, pp. 288-303. Springer, Heidelberg (2002) 